\title{
A New Approach to Adaptive Power Line Interference Removal
}

\author{
Abhay R. Kasetwar ${ }^{1}$ and Sanjay M. Gulhane ${ }^{2}$ \\ ${ }^{1}$ Department of Electronics and Telecommunication Engineering, \\ Bapurao Deshmukh College of Engineering, Sevagram, Wardha, Maharashtra, India. \\ abhaykasetwar@gmail.com \\ ${ }^{2}$ Department of Electronics and Telecommunication Engineering, \\ Jawaharlal Darda Institute of Engineering \& Technology, Yavatmal, Maharashtra, India. \\ smgulhane67@rediffmail.com
}

\begin{abstract}
Many studies have been devoted to the adaptive power line interference (PLI) removal design for biomedical signal processing. Most of the existing PLI removal methods are developed for applications in which the presence of PLI strongly affects the system performance. The objective of the work is to investigate the suitable method that is more feasible for its FPGA implementation. The detail study is made on available methods for PLI removal for biomedical signal processing. Different algorithms like LMS, NLMS, SLMS etc. have been analyzed for their applications in adaptive power line interference removal. LMS algorithm with some modifications in weight updation process is proposed for better performance of adaptive power line interference removal. The weights at which system got minimum error in the output are chosen using bubble sorting method to recover clean ECG signal. The implementation of adaptive PLI removal using Minimum Error Weight Adjustment (MEWA) made the system more efficient in terms of signal to noise ratio (SNR) and correlation coefficient with less computational complexity. In FPGA implementation of the system, the focus will be on making the implementation efficient in area and power utilization by using different area and power minimization techniques.
\end{abstract}

Keywords: ECG; PLI; adaptive filtering; LMS; MEWA; FPGA

\section{Motivation and Problem Identification}

An ECG signal is basically an index of the functionality of the heart. For example, a physician can detect arrhythmia by studying abnormalities in the ECG signal. Since very fine features present in an ECG signal may carry important information, it is necessary to have the signal as clean as possible. But all ECG capturing machines require ac supply due to which power line interference $(50 / 60 \mathrm{~Hz})$ is getting intro-duced in the ECG which distorts the signal badly. The fixed notch filter cannot be the solution because frequency of power line varies about fractions of a Hertz, or even a few Hertz. So we have to use the adaptive filter for the removal of such nonstationary noise. Many 
algorithms have been proposed for the implementation of same but could not achieve the best suited algorithm which can give the solution for frequency, amplitude and phase variations simultaneously without distorting the characteristics of original ECG signal.

So it is required to implement novel method for eliminating power line interference using adaptive filter without distorting the original biomedical signal.

\section{Introduction}

One of the application areas of signal processing techniques is biomedical engineering such as electrocardiography. The electrocardiogram (ECG) is graphical representation of cardiac activity of heart. An ECG signal is an index of functionality of heart which plays very important role in the diagnosis of heart diseases. Very fine features in ECG signal carry important information regarding patient's heart condition. So it is very necessary to have a noise free ECG for correct diagnosis. Most of the medical equipments used in hospitals are powered by power supply of working frequency $50 / 60 \mathrm{~Hz}$. American Heart Association recommends that ECG signal recorder have $3 \mathrm{~dB}$ frequency range from $0.67 \mathrm{~Hz}$ to $150 \mathrm{~Hz}$. As power supply frequency lies within the frequency range of ECG signal, measured ECG signal is corrupted by power line interference (PLI) [2]. PLI with low frequency and weak amplitude may totally mask the signal of interest and affects the reliability and accuracy of ECG signal. For the quality analysis of cardiac diseases, PLI should be removed from ECG signal, while keeping ECG signal intact [4]. Traditional approach to remove PLI was to use a notch filter with narrow rejection bandwidth. Increase in attenuation level of notch filter, PLI can be removed effectively however increased bandwidth disturbs the nearby spectrum. This is the major drawback of using fixed notch filter for removal of PLI from ECG signal [2]. This approach is not suitable when PLI is non-stationary in nature, i.e. when frequency, phase and amplitude of PLI are varying with respect to time. So adaptive interference canceller is required for the removal of such kind of interference. Adaptive filters have the capability of tracking and detecting dynamic variations and time varying potentials. The purpose of an adaptive interference canceller is to subtract non-stationary type of noise from a received signal in adaptively controlled manner so as to improve the signal to noise ratio (SNR). In adaptive PLI cancellation method, PLI is estimated by minimizing difference using adaptive algorithms. Further estimated PLI is subtracted from noisy ECG signal to cancel the PLI at successive iteration [7]. The adaptive interference canceller shown in Fig. 1 operates on the reference PLI input $\mathrm{i}(\mathrm{n})$ to produce estimate of the noise which is then subtracted from the desired signal $\mathrm{d}(\mathrm{n})$, i.e. noisy signal to get the error signal e(n). Further the weight updation is done by MEWA LMS algorithm to minimize the error.

Power line interference may occur through two mechanisms: capacitive and inductive coupling. In capacitive coupling, the transfer of energy between two circuits by means of a coupling capacitance pre-sent between two circuits. The value of the coupling capacitance decreases with increasing separation of the circuits while inductive coupling causes because of mutual inductance between two conductors. The geometry of the conductors as well as the separation between them determines the value of the 


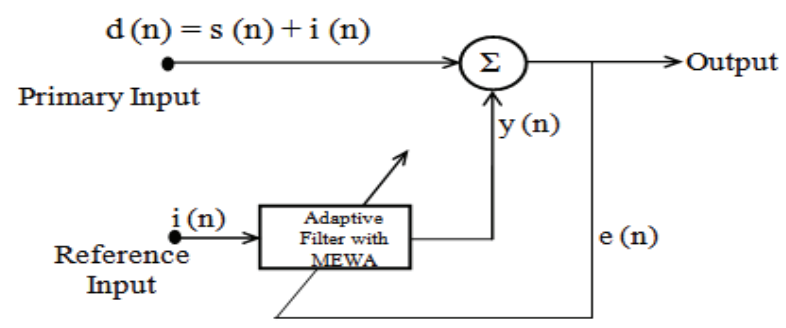

Fig. 1. Adaptive Interference Canceller.

mutual inductance, and hence the degree of inductive coupling. Actually, capacitive coupling is responsible for high frequency interference while inductive coupling causes low frequency interference. For this reason, inductive coupling is the dominant mechanism of power line interference in electrocardiography. Ensuring the proper application of electrodes, that there are no loose wires, and all components have adequate shielding which can help to minimize occurrence of amount of power line interference.

\section{Related Work and Preliminaries}

Author presents a survey on implementation of an adaptive power line interference canceller for ECG signal processing and recommends to use Least mean-square (LMS) algorithm. Notch filters are ineffective, whenever the power line frequency is not stable or not accurately known. A mismatch between the suppression band and the power line frequency might lead to inadequate reduction of power line interference therefore adaptive interference cancellers should be preferred. LMS algorithm is commonly used for adaptive filtering since it is computationally simple and efficient [1]. The least mean square (LMS) is the most used algorithm to iteratively minimizing the mean square error (MSE) of the system output. In some practical applications, the LMS algorithm can be implemented only with delayed coefficient adaptation [11]. The use of delayed coefficient adaptation in the LMS algorithm has enabled the design of modular systolic architectures [12]. The convergence behavior of this delayed least mean squares (DLMS) algorithm, when compared with that of the standard LMS algorithm, its performance is degraded with the increase in the adaptation delay. Modular design systolic architecture for transversal adaptive filtering that maintains the convergence behavior of the LMS algorithm by minimizing the adaptation delay, and also supports high input sampling rates with minimal input/output latency [15]. The first used adaptive method to remove $50 / 60 \mathrm{~Hz}$ interference from a corrupted ECG signal using adaptive filter was proposed by Widrow. This method is very robust and has the capability to remove $50 / 60 \mathrm{~Hz}$ interference [8]. Ziarani et al. proposed nonlinear adaptive filter for removal of PLI from ECG signal. Structure of filter is highly simple and required little arithmetic [5].

Adaptive noise canceller (ANC) method with internal reference signal is introduced by Ziarani which is having more complex structure. Martenes et al. proposed simple adaptive noise canceller as improvement in Widrow method and neglecting the presence of PLI harmonics [6]. Ziarani proposed very practical method for PLI removal but 
adaptation constant may not lead to a successful equation phase. The im-proved adaptive canceller (IAC) is proposed by Martens et al. with important improvement such as error filtering and adaption blocking based on QRS complex detection [7].

Further the work is extended by Bharath with the use of appropriate window length. Yue Der Lin and Yu Hen Hu proposed LDA based adaptive filter overcome drawback of unrealistic assumptions for existing methods with less computational complexity. Structure is capable to eliminate PLI with variable frequency and other sinusoidal interference [3]. Some researchers have presented the real time imple-mentation of an adaptive canceller using FPGA. Larger the value of step size parameter (u) increases the convergence speed but affects the stability and vice versa [14],[17].

\section{Proposed Method}

A block diagram of the proposed adaptive PLI removal using Minimum Error Weight Adjustment (MEWA) is depicted in Figure 1. This proposed adaptive canceller is capable to suppress the nonstationary PLI in ECG recordings. Weights of filter are automatically updated using MEWA algorithm. This algorithm is highly efficient because of its robustness and low computational complexity. We have used the sliding window approach for filtering. As the order of filter is 2, the window length is also taken as 2. So the computational complexity is getting reduced which will be a very important parameter for real time implementation. The weights at which the system got minimum error in the output are chosen to recover the clean ECG signal. The first order digital FIR filter is used for filtering purpose. Both the filter weights are getting updated at every iteration. The performance of system is analyzed by observing the value of error for different values of step size parameter and number of filter taps. The bubble sorting method has been used to find out the location at which minimum value of error is present. That location identifies the most appropriate weights of filter to reconstruct the required ECG signal. Those weights are maintained till the next minimum value of error gets appear. So the method is called as Minimum Error Weight Minimization (MEWA) Method. Sign Least Mean Square (SLMS) algorithm is used for updating the weights. Different PLI signals are artificially generated for testing purpose using inductive coupling mechanism. Mathematically, MEWA can be demonstrated as follows:

$$
e(n)=d(n)-y(n)
$$

where $\mathrm{d}(\mathrm{n})=\mathrm{x}(\mathrm{n})+\mathrm{i}(\mathrm{n})$

$x(n)$ is a clean ECG signal and $i(n)$ is added interference

$$
\begin{gathered}
\mathrm{W}=\operatorname{lam} * \mathrm{~W}+\mathrm{mu}^{*} \operatorname{sign}(\mathrm{e}(\mathrm{n}))^{*} \mathrm{X} \text {; where } \mathrm{e}(\mathrm{n}) \text { is an error signal } \\
\operatorname{Location}(\mathrm{l})=\mathrm{n}\{\min (\mathrm{e}(\mathrm{n}))\}
\end{gathered}
$$

$$
\mathrm{w}=\mathrm{w}(\mathrm{l}) \text {; where } \mathrm{w} \text { is the current weight vector }
$$




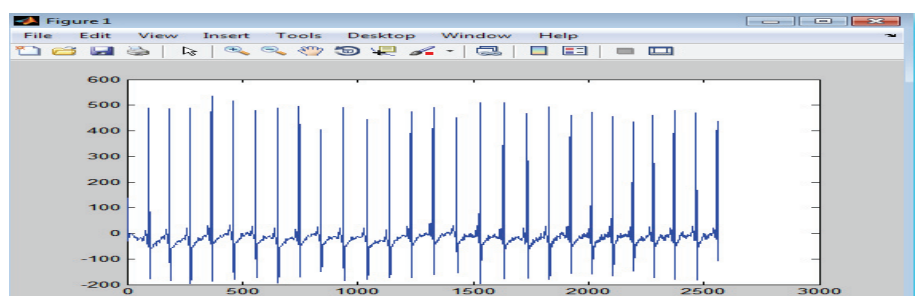

Fig. 2. Clean ECG signal from MITBIH database.

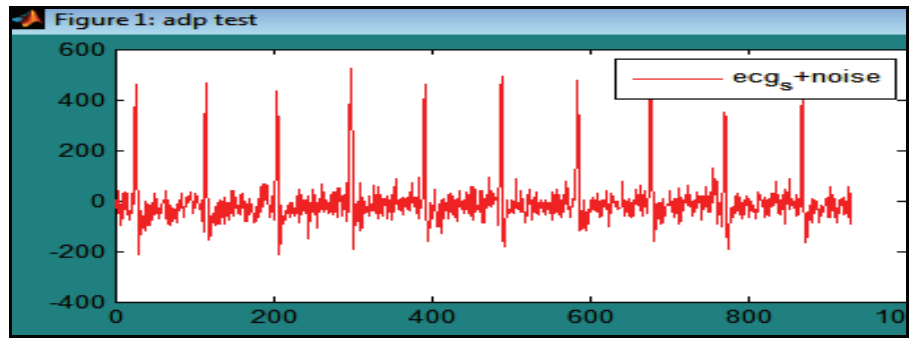

Fig. 3(a). Noisy ECG Signal with 5 db SNRin.

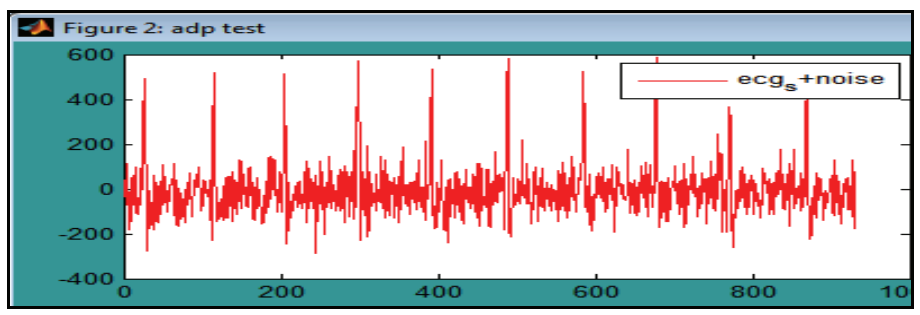

Fig. 3(b). Noisy ECG Signal with 0 db SNRin.

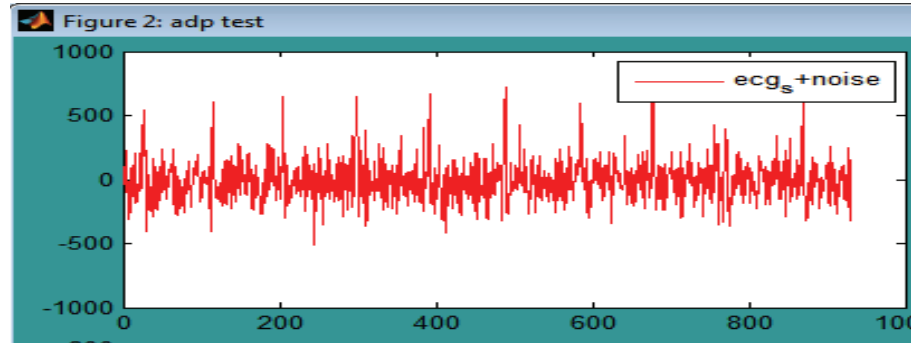

Fig. 3(c). Noisy ECG Signal with -5 db SNRin.

\subsection{Evaluation Method}

In order to validate the performance of MEWA LMS algorithm, simulation is carried out using ECG signals from MITBIH database as shown in Fig. 2. The ECG signals are of $1 \mathrm{~s}$ duration with a sampling frequency of $250 \mathrm{~Hz}$. First the signal is passed 
through a channel to introduce channel noise. Further the received ECG is added with the additive noise to form a noisy signal as shown in Fig. 3(a)(b)(c). This signal is used as an input signal to evaluate the performance. To quantify the performance of the interference canceller, signal to noise ratio (SNR) and the correlation coefficient (CC) between the original ECG signal and the filtered ECG signal are calculated. Signal to noise ratio is measured at the input, as SNRin and at the output as SNRout of the adaptive canceller. SNR improvement is one of the most important parameter to evaluate the system performance. SNRin is defined as the ratio of the power of the ECG signal to the power of added noise and SNRout is defined as the ratio of the power of the ECG signal to the residual noise power. And Correlation Coefficient gives the degree of similarity between clean ECG and filtered ECG.

\subsection{Results and Discussion}

Clean ECG signal recorded at Beth Israel Hospital (BIH) in Boston and made available by Massachusetts Institute of Technology (MIT) is taken for simulation. The clean ECG signal is having 2560 discrete values as shown in Fig. 2. This clean ECG signal is passed through noisy channel and further polluted by synthetically generated nonstationary interference as shown in Fig. 3 (a)(b)\&(c). The algorithm is analyzed by using MATLAB simulation. In order to compare the performance of proposed adaptive algorithm, first the performance of normal LMS algorithm is taken into the consideration. Thereafter proposed MEWA LMS algorithm is analyzed for different values of step size parameter. Following Table 1 shows the comparative analysis of normal LMS algorithm with MEWA LMS algorithm with respect to SNRout and Corr. Coeff values.

Fig. 4 (a)(b)(c) shows the simulation results of MEWA LMS algorithm for improved values of SNRout and Correlation Coefficient (CC) when $\mathrm{u}=0.01$ and No. of taps $=2$. In each Fig. plot 1 shows the overlapping of noisy and recovered noisy signal where plot 2 shows the overlapping of clean ECG and and recovered ECG signal.

Table 1. SNRout and Corr. Coeff. Values for Normal LMS and MEWA LMS.

\begin{tabular}{|c|c|c|c|c|c|}
\hline \multirow{2}{*}{$\begin{array}{c}\text { No.of } \\
\text { taps } \\
(\mathbf{N})\end{array}$} & \multirow{2}{*}{$\begin{array}{c}\text { SNRin } \\
(\mathbf{d b})\end{array}$} & \multicolumn{2}{|c|}{ SNRout(db) } & \multicolumn{2}{c|}{ Corre. Coeff.(CC) } \\
\cline { 3 - 6 } & & $\begin{array}{c}\text { Normal LMS } \\
(\mathbf{u}=\mathbf{0 . 0 0 0 0 0 1})\end{array}$ & $\begin{array}{c}\text { MEWA } \\
\text { LMS } \\
(\mathbf{u = 0 . 0 1})\end{array}$ & $\begin{array}{c}\text { Normal } \\
\text { LMS }\end{array}$ & $\begin{array}{c}\text { MEWA } \\
\text { LMS }\end{array}$ \\
\hline 2 & -10 & 1.34 & 32.83 & 0.6535 & 0.9833 \\
\hline 2 & -5 & 7.82 & 37.2 & 0.6457 & 0.9816 \\
\hline 2 & 0 & 13.39 & 41.82 & 0.6695 & 0.9832 \\
\hline 2 & 5 & 18.62 & 44.04 & 0.8044 & 0.9815 \\
\hline
\end{tabular}




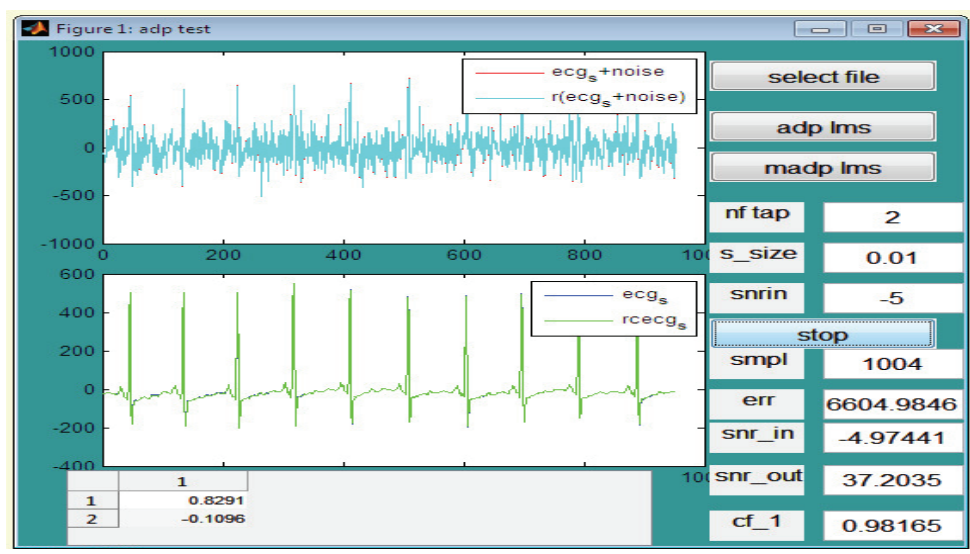

Fig. 4(a). Output of MEWA LMS for SNRin $=-5 \mathrm{db}$.

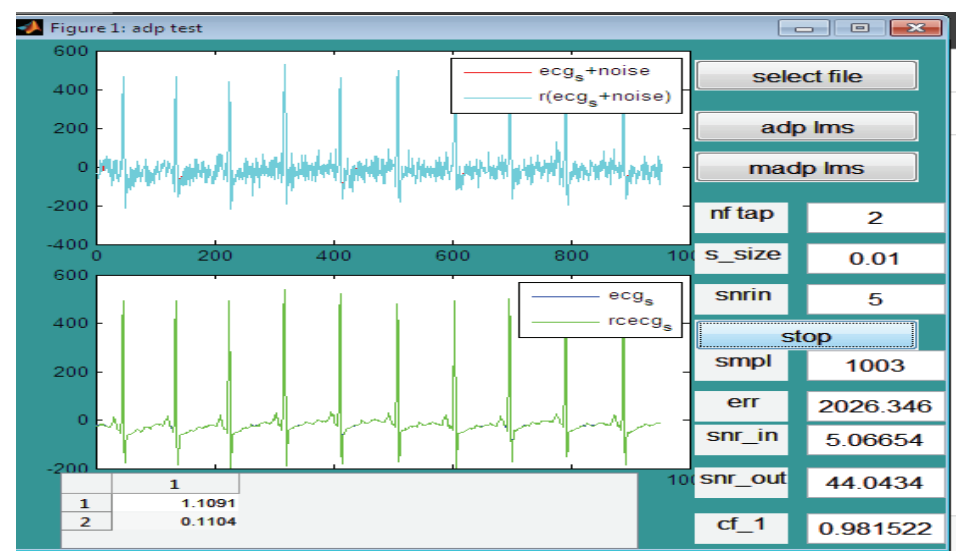

Fig. 4(b). Output of MEWA LMS for SNRin $=0 \mathrm{db}$.

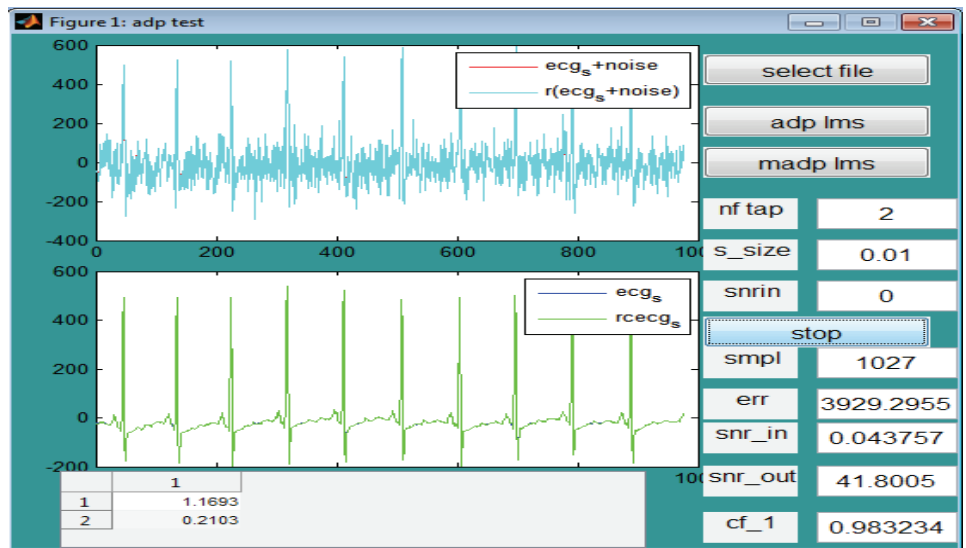

Fig. 4(c). Output of MEWA LMS for SNRin $=5 \mathrm{db}$. 


\section{Conclusion}

Cancellation of Power Line Interference from ECG signal is a challenging problem as the frequency of Power Line Interference may change with time. This work suggested the minimum error weight adjustment (MEWA) mechanism for LMS algorithm. An adaptive interference canceller using MEWA LMS efficiently filters the ECG signal without distorting the characteristics of ECG signal with fewer computations. The choice of step size parameter is again a critical issue. Result shows the improved values of SNRout and Correlation coefficient. As the filter requires only two taps for designing, the computational complexity is getting reduced. This will help the designer for real time implementation. The proposed algorithm gives SNR improvement of about $40 \mathrm{db}$. The comparative analysis proves the advantage of MEWA LMS over Normal LMS.

\section{Work in Progress}

Currently the work is going on for finding the best suitable algorithm for adaptive power line interference removal. The feasibility of algorithm for real time implementation will be checked first. Once the algorithm is getting finalized, real time implementation of the algorithm will be done. Adaptive interference cancellers can be implemented using general-purpose microcontrollers or digital signal processors. These solutions are not optimum since they have low processing speed and sequential execution due to the fact that adaptive cancellers are implemented with a software program. Another solution is the implementation of the adaptive controller with field-programmable gate array (FPGA) [10][19].

The adaptive filters can be implemented by sequential, parallel and semi-parallel architectures. The type of architecture chosen is based on sample rate and number of coefficients. The parallel architecture is well suited for a high sampling rate requirements and a small number of coefficients. However, the sequential architecture is more suited for the low sampling rate requirements and a large number of coefficients. The semi-parallel architecture is a good compromise that permits to implement filters having a large number of coefficients and requiring a high sampling rate [9]. So the adaptive power line interference removal will be implemented using semi-parallel architecture on FPGA.

After real time implementation on FPGA, the next target is to calculate the requirement of power and area for chip level implementation probably on 90nm technology. Quartus and Cadence tool will be used for detail power and area analysis.

\section{References}

1. Abhay R. Kasetwar and Sanjay M. Gulhane : "Adaptive Power Line Interference Can-celler A Survey" in International Journal of Advances in Engineering \& Technology, Jan. 2013 ISSN: 2231-1963.

2. Maryam Butt, Nauman Razzaq, Ismail Sadiq.: "Power Line Interference removal from ECG Signal using SSRLS Algorithm" $9^{\text {th }}$ International Colloquium on Signal Processing and Applications, 2013. 
3. Yue-Der Lin, Yu Hen Hu: "Power Line Interference Detection and Suppression in ECG Signal Processing," IEEE Transactions on Biomedical Engineering, Vol.55, No.1 January2008.

4. H. N. Bharat, K. M. M. Prabhu: "A New LMS based Adaptive Interference Canceller for ECG Power Line Removal," International Conference on Biomedical Engineering February, 2012.

5. Alireza K. Ziarani, Adalbert Konrad: "A Nonlinear Adaptive Method of Elimination of Power Line Interference in ECG Signals," IEEE Transactions on Biomedical Engineering, Vol. 49, No. 6 June 2002.

6. Martens S.M.M., Bergmans J.W.M. and Oei S.G.: "A Simple adaptive interference cancellation method for power line reduction in electrocardiography", proceeding of $25^{\text {th }}$ Symposium on Information Theory in the Benelux, pp. 49-56 (2004).

7. Martens S.M.M., Mischi M., Oei S.G, and Bergmans J.W.M.: “An improved adaptive power line interference canceller for electrocardiography", IEEE Transactions on Biomedical Engineering, Vol.53, pp. 2220-2231 (2006).

8. B. Widrow et al :"Adaptive noise cancelling : principles and application." Proceeding of the IEEE, Vol. 63, no. 12, pp.1692-1716. Dec. 1975.

9. Abhay R. Kasetwar and Dr. Sanjay M. Gulhane: "A Survey of FPGA Based Interference Cancellation Architectures for Biomedical Signals" in International Conference on Computer Communication and Informatics (ICCCI-2013), Jan. 04-06, 2013, Coimbatore,INDIA 978-1-4673-2907-1/13/\$31.00@2013IEEE.

10. Mohammed Bahoura, et al.: "FPGA-Implementation of a Sequential Adaptive Noise Canceller using Xilinx System Generator," in International Conference on Microelectronics, 2009.

11. G. Long, F. Ling, and J. Proakis: "LMS algorithm with delayed coefficient adaptation," IEEE Transaction on Acoustic, Speech \& Signal Processing, vol. 37, no. 9, pp. 1397-1405, 1989.

12. Dai Huhe, et al:: "A Novel Suppression Algorithm of Power Line Interference in ECG Signal," in First International Conference on Pervasive Computing, Signal Processing and Applications, 2010.

13. B. S. Lin et al.: "Removing residual power-line interference using WHT adaptive filter," in Proceedings of Second Joint EMBS / BMES Conference, USA, Oct 23 - 26, 2002.

14. LD Avendano-Valencia, et al.: "Improvement of an Extended Kalman Filter Power Line Interference Suppressor for ECG Signals," in Journal of Computer in Cardiology, 2007, vol. 34 , pp. 553 - 556G.

15. Ramanathan S, et al.: "A systolic architecture for LMS adaptive filtering with minimal adaption delay," in Proceedings of $9^{\text {th }}$ International Conference on VLSI Design, Jan. 1996, pp $286-289$.

16. Zhiguo Zhou, et al:: "FPGA-Implementation of LMS Adaptive Noise Canceller for ECG Signal Using Model Based Design," in IEEE International Symposium on Bioelectronics and Bioinformatics, Nov 3-5, 2011.

17. N. Jindapetch, S. Chewae, P. Phukpattaranont: "FPGA implementation of an ADALINE adaptive filter for power line noise cancellation in surface electromyography signals" in ELSEVIER measurement, 45 (2012) 405-414.

18. G. Chen : "The FPGA Implementation of Kalman Filter," in 5th WSEAS nternational Conference on Signal Processing, Computational Geometry \& Artificial Vision, 2005.

19. R. Ramos et al., "Application by means of FPGA of an adaptive canceller $50 \mathrm{~Hz}$ interference in electrocardiography," in IEEE Instrumentation and Measurement Technology Conference, Hungary, May 21-23, 2001. 